- Original Article

\title{
Sarcopenia Is Not Associated with Depression in Korean Adults: Results from the 2010-2011 Korean National Health and Nutrition Examination Survey
}

\author{
Chae-Hwa Byeon', Kee-Young Kang', Se-Hun Kang', Han-Kyul Kim², Eun-Jin Bae ${ }^{1, *}$ \\ ${ }^{1}$ Department of Family Medicine, Daedong Hospital, Busan, Korea \\ ${ }^{2}$ Department of Family Medicine, Dong-A University Hospital, Busan, Korea
}

Background: Sarcopenia is associated with metabolic disorders, cardiovascular disease, and mortality; however, its association with depression in the general population remains unknown. Therefore, we investigated this association in Korea.

Methods: This study included 8,958 and 8,518 subjects from the 2010-2011 Korean National Health and Nutrition Examination Survey V-1, 2. The study was restricted to participants $\geq 20$ years of age who had completed the survey, including whole-body dual-energy X-ray absorptiometry scans. After exclusion, 7,364 subjects were included in our final analysis. Age was categorized into three groups (20-39, 40-59, and $\geq 60$ years), and subjects were categorized according to their sarcopenic and obesity status. Depression was categorized into three groups (not depressed, depressed, and depression).

Results: The sarcopenia group did not have a higher prevalence of depression or depressive symptoms compared to the nonsarcopenia group; the same was true even when obesity was considered. All age groups showed non-significant associations between sarcopenia and depression. In multivariate logistic regression models, no significant associations were observed between sarcopenia and prevalence of depression or depressed symptoms in men and women.

Conclusion: We found no associations between sarcopenia and the prevalence of depression or depressed symptoms in Korean adults. Future large prospective studies and randomized controlled trials are needed to further assess this relationship.

Keywords: Sarcopenia; Depression; Aging 


\section{INTRODUCTION}

Depression is a mood disorder with symptoms that include persistently depressive mood, loss of motivation or interest, and feelings of guilt. Severe cases may be accompanied by eating and memory disorders, and may also lead to suicide. ${ }^{1)}$ The data from the 2005-2008 National Health and Nutrition Examination Survey conducted in the United States reported that approximately $21.6 \%$ of participants showed depressive symptoms ${ }^{2)}$ whereas the World Health Organization World Mental Health (2004) Surveys reported lifetime prevalences of major depressive disorders of $19.2 \%, 21.0 \%$, and $17.9 \%$ for the United States, France, and the Netherlands, respectively. ${ }^{3)}$ Based on the 2011 Epidemiological Survey of Mental Disorders in Korea from the Ministry of Health and Welfare, $6.7 \%$ of participants 18 years and older experienced major depressive disorders at least once in their lifetimes. ${ }^{4}$ The prevalence of major depressive disorders in Korea is lower than that reported in other countries. This observation is interpreted as a sociocultural difference, in that Far East Asian cultures do not satisfy the diagnostic criteria for major depressive disorders: assessments often reveal no major symptoms because the individuals themselves consider depressive symptoms to be mild conditions. ${ }^{5)}$

In 1988, Rosenberg ${ }^{6)}$ first proposed the term 'sarcopenia.' Sarcopenia is an aging-associated condition defined as the loss of muscle strength due to loss of muscle mass. The diagnostic criteria for sarcopenia were presented by Baumgartner et al. ${ }^{7)}$ in the 1998 New Mexico Elder Health Survey, but there is currently no generally accepted diagnostic method for sarcopenia. Studies on sarcopenia have made comparisons to osteoporosis, another typical physical change in the elderly, but studies of osteoporosis are lacking. ${ }^{8)}$ Janssen et al. ${ }^{9)}$ reported that the social cost associated with sarcopenia in the United States reached $\$ 18.5$ billion in 2000 , and noted the need for measures to address sarcopenia. The need for studies on and measures for sarcopenia is apparent in Korea as well, as it becomes an increasingly aging society.

Sarcopenia is associated with physical inactivity, disability, diabetes, metabolic syndrome, cardiovascular diseases, and mortality. ${ }^{10,11)}$ Since depression is also linked to physical inactivity or dysfunction, ${ }^{12)}$ there may be an association between sarcopenia and depression. The relationship between depression and obesity is well known. Meta-analyses have also the high probability that obesity accompanies and may be a risk factor for depression. ${ }^{13)}$ In a study on sarcopenic obesity and mental health, Cho et al. ${ }^{14)}$ also reported that sarcopenic obesity was associated with anxiety and depressive symptoms. Therefore, we speculated that sarcopenia and sarcopenic obesity would be associated with depression.

While recent studies have evaluated the association between sarcopenia and physical symptoms and diseases, studies on the association with mental health, including depression, are lacking. Although Kim et al. ${ }^{15)}$ and Hsu et al. ${ }^{16)}$ demonstrated that sarcopenia is associated with depression, additional studies are needed.

Studies on sarcopenia and depression to this point have been insufficient and few have included younger age groups. Therefore, the present study used data from the 2010-2011 Korean National Health and Nutrition Examination Survey (KNHANES $\mathrm{V}-1,2)$ to examine the relationship between sarcopenia and depression in the Korean population by age group and obesity status.

\section{METHODS}

\section{Study Population}

The KNHANES is conducted annually using a systematic sampling method after stratifying for population ratios based on the town units of dong, eup, and myun, as well as housing type and age group. The survey participants include household members at least one year of age from approximately 3,800 households nationwide. The first year of the fifth survey was conducted between January and December 2010; the second year continued from January to December 2011. ${ }^{17)}$ The present study used data from 2010 and 2011; the most recent data that also included dual energy X-ray absorptiometry (DEXA). The present study included only those participants 20 years or older who had completed both the DEXA and the questionnaire. Participants with physician-diagnosed diseases that could also affect depression, such as cancer, liver cirrhosis, kidney failure, or cerebro- or cardiovascular diseases, were also excluded. The total study population was 7,364 individuals, comprised 5,245 and 2,119 people from 2010 and 2011, respectively, after excluding 1,420 and 4,381 of 6,665 and 6,500 adults for missing values. The present study used data from the KNHANES-V, which received institutional review board approval (2010-02CON-21-C, 2011-02CON-06-C).

\section{Measurement}

\section{1) Depressed state}

As described previously, ${ }^{18)}$ patients were classified as having depression, depressed symptoms, or not depressed. The depression group comprised respondents who indicated that they had been clinically diagnosed with depression. Among those who had not been clinically diagnosed as having depression, those who responded "yes" to the question "In the past year, have you felt sadness or despair continuously for two or more weeks that was severe enough to interfere with daily life?" were assigned to the depressed symptom group. Similarly, those who were not clinically diagnosed as having depression and who also did not report having experienced depressive symptoms for 2 weeks or more in the past year were categorized as 
not depressed.

\section{2) Obesity and sarcopenia}

Obesity was diagnosed using body mass index (BMI), with BMI $\geq 25$ defined as obese when height ( $\mathrm{Ht}$ ) and weight $(\mathrm{Wt})$ were substituted into the equation, $\mathrm{BMI}=\mathrm{Wt} / \mathrm{Ht}^{2}$.

Sarcopenia was diagnosed using a modified version of a method described in a previous study. ${ }^{19)}$ After measuring participant appendicular skeletal muscle mass (ASM) via DEXA, the ASM/Wt (\%) values were calculated; participants with values two standard deviations below the mean of the younger age group were classified as having sarcopenia. ${ }^{20)}$ The mean ASM/ Wt (\%) of participants $20-39$ years of age, $26.88 \%$ for men and
$21.02 \%$ for women, were used as the cut-off points. Depending on their sarcopenia and obesity status, the study participants were divided into sarcopenic obese, nonsarcopenic obese, sarcopenic nonobese, and nonsarcopenic nonobese groups.

\section{3) Metabolic syndrome}

Metabolic syndrome was diagnosed using the modified adult treatment panel III presented in 2005 by the American Heart Association and National Heart, Lung, and Blood Institute. ${ }^{21)}$ Patients satisfying three or more of the five criteria listed below were considered to have metabolic syndrome: (1) waist size $\geq 90 \mathrm{~cm}$ for men and $\geq 85 \mathrm{~cm}$ for women; ${ }^{22)}$ (2) triglyceride levels over $150 \mathrm{mg} / \mathrm{dL}$ or under treatment for hyperlipidemia; (3)

Table 1. Study population characteristics according to sarcopenic status

\begin{tabular}{|c|c|c|c|c|}
\hline Characteristic & Total & Sarcopenic & Nonsarcopenic & P-value* \\
\hline Total & $7,364(100.0)$ & 319 (3.6) & $7,045(96.4)$ & $<0.001$ \\
\hline Age (y) & $49.48 \pm 0.54$ & $54.71 \pm 0.97$ & $44.24 \pm 0.37$ & $<0.001$ \\
\hline $20-39$ & $2,341(40.6)$ & $39(20.0)$ & $2,302(41.4)$ & $<0.001$ \\
\hline $40-59$ & $2,899(41.8)$ & 99 (39.6) & $2,800(41.9)$ & $<0.001$ \\
\hline$\geq 60$ & $2,124(17.6)$ & $181(40.4)$ & $1,943(16.8)$ & $<0.001$ \\
\hline Men & $3,187(50.1)$ & $109(37.2)$ & $3,078(50.6)$ & $<0.001$ \\
\hline Women & $4,177(49.9)$ & $210(62.8)$ & $3,967(49.4)$ & $<0.001$ \\
\hline Body height (cm) & $161.60 \pm 0.31$ & $158.73 \pm 0.59$ & $164.46 \pm 0.17$ & $<0.001$ \\
\hline Body weight (kg) & $67.31 \pm 0.52$ & $70.70 \pm 1.03$ & $63.92 \pm 0.20$ & $<0.001$ \\
\hline Body mass index $\left(\mathrm{kg} / \mathrm{cm}^{2}\right)$ & $25.71 \pm 0.15$ & $27.89 \pm 0.29$ & $23.54 \pm 0.06$ & $<0.001$ \\
\hline Waist circumference (cm) & $86.67 \pm 0.37$ & $92.64 \pm 0.72$ & $80.70 \pm 0.18$ & $<0.001$ \\
\hline ASM (kg) & $17.24 \pm 0.17$ & $15.87 \pm 0.33$ & $18.62 \pm 0.09$ & $<0.001$ \\
\hline ASM/body weight (\%) & $25.54 \pm 0.10$ & $22.18 \pm 0.19$ & $28.91 \pm 0.08$ & $<0.001$ \\
\hline Fasting plasma glucose (mg/dL) & $100.76 \pm 1.02$ & $105.47 \pm 2.06$ & $96.05 \pm 0.36$ & $<0.001$ \\
\hline Total cholesterol (mg/dL) & $193.42 \pm 1.37$ & $199.82 \pm 2.62$ & $187.02 \pm 0.59$ & $<0.001$ \\
\hline Triglyceride (mg/dL) & $146.56 \pm 3.77$ & $160.85 \pm 7.42$ & $132.27 \pm 1.93$ & $<0.001$ \\
\hline High density lipoprotein cholesterol (mg/dL) & $47.65 \pm 0.45$ & $46.64 \pm 0.89$ & $48.66 \pm 0.18$ & $<0.001$ \\
\hline Low density lipoprotein cholesterol (mg/dL) & $116.46 \pm 1.22$ & $121.02 \pm 2.34$ & $111.90 \pm 0.54$ & $<0.001$ \\
\hline Systolic BP (mm Hg) & $123.01 \pm 0.64$ & $127.65 \pm 1.23$ & $118.36 \pm 0.35$ & $<0.001$ \\
\hline Diastolic BP (mm Hg) & $78.64 \pm 0.43$ & $80.28 \pm 0.80$ & $77.00 \pm 0.23$ & $<0.001$ \\
\hline Metabolic syndrome & $2,211(26.5)$ & $195(58.7)$ & $2,016(25.3)$ & $<0.001$ \\
\hline Depression status & & & & 0.171 \\
\hline Not depressed & $6,293(85.5)$ & $266(81.1)$ & $6,027(85.7)$ & \\
\hline Depressed & $793(10.9)$ & $36(13.8)$ & $757(10.8)$ & \\
\hline Depression & $278(3.6)$ & $17(5.1)$ & $261(3.5)$ & \\
\hline Smoking status ${ }^{\dagger}$ & & & & 0.302 \\
\hline Never & $4,283(52.6)$ & $197(55.4)$ & $4,086(52.4)$ & \\
\hline Former & $1,513(20.3)$ & $74(22.5)$ & $14.39(20.3)$ & \\
\hline Current & $1,568(27.1)$ & $48(22.0)$ & $1,520(27.3)$ & \\
\hline Regular exercise ${ }^{\ddagger}$ & & & & 0.211 \\
\hline Yes & $3,541(49.6)$ & $136(44.8)$ & $3,405(49.8)$ & \\
\hline No & $3,823(50.4)$ & $183(55.2)$ & $3,640(50.2)$ & \\
\hline Menopause status (women) & & & & $<0.001$ \\
\hline Natural & $1,722(16.3)$ & $147(36.1)$ & $1,575(15.6)$ & \\
\hline Surgical & $260(2.7)$ & $17(6.1)$ & $243(2.5)$ & \\
\hline No & $5,382(81.0)$ & $155(57.8)$ & $5,227(81.9)$ & \\
\hline
\end{tabular}

Values are presented as mean \pm standard error of number (\%).

ASM, appendicular skeletal muscle mass; BP, blood pressure.

${ }^{*}$ Calculated by analysis of variance and chi-square tests. ${ }^{\dagger}$ Smoking status was divided into three categories: never, former, and current smoker. *Regular exercise was defined as "yes" when the participant performed moderate or strenuous exercise on a regular basis (for more than 30 minutes at a time and more than five times per week in the case of moderate exercise; for more than 20 minutes at a time in the case of strenuous exercise) or when the subject walked for more than 30 minutes at a time and more than five times per week. Strenuous exercise included running, climbing, bike riding at fast speed, swimming at fast speed, football, basketball, jumping rope, squash, tennis of singles, and physical activities such as carrying heavy objects. Moderate exercise included swimming at slow speed, tennis of doubles, volleyball, badminton, ping-pong, and physical activities such as carrying light objects. 
blood pressure $(\mathrm{BP}) \geq 130 / 85 \mathrm{~mm} \mathrm{Hg}$ or under treatment for hypertension; (4) high density lipoprotein cholesterol levels (HDLC) $<40 \mathrm{mg} / \mathrm{dL}$ for men and $<50 \mathrm{mg} / \mathrm{dL}$ for women; and (5) fasting blood glucose levels over $100 \mathrm{mg} / \mathrm{dL}$ or under treatment for diabetes.

Serum triglyceride, HDLC, and fasting blood glucose levels were measured via an enzymatic method using a Hitachi Automatic Analyzer 7600 (Hitachi, Tokyo, Japan).

\section{Statistical Analysis}

Because the number of subjects in the KNHANES varies from year to year, integrated weighted values were used for the analysis. Chi-square tests and analysis of variance were used to determine if the presence of sarcopenia was significantly associated with differences in depressed state. Stratified analysis was used to examine the association between sarcopenia and depression by age groups, while multiple logistic regression analysis was adjusted for covariates and performed to determine the relationship between sarcopenia and depression by gender. Gender, age, BMI, smoking, metabolic syndrome, diabetes, hypertension, and menopause (in women) were used as covariates. The statistical significance was set to $<0.05$. Categorical and continuous data were expressed as frequencies and means \pm standard error. IBM SPSS Statistics for Windows ver. 22.0 (IBM Co., Armonk, NY, USA) was used for all statistical processing.

\section{RESULTS}

\section{Clinical Characteristics of the Study Population}

The mean ages in the sarcopenia and nonsarcopenia groups were 54.71 and 44.24 years, respectively, indicating a higher mean age among participants in the sarcopenia group. The sarcopenia group had higher total cholesterol, triglyceride, and low density lipoprotein cholesterol (LDLC) levels, but lower HDLC than the nonsarcopenia group. The prevalence of metabolic syndrome in the sarcopenia and nonsarcopenia groups was $58.7 \%$ and $25.3 \%$, respectively. However, the difference in the prevalence of depressive symptoms and depression with sarcopenia between these groups was not statistically significant (Table 1).

\section{Depression by Sarcopenia and Obesity Status}

The prevalence of sarcopenia in the obese and nonobese groups was $8.6 \%$ and $1.2 \%$, respectively. Participants with sarcopenia who were also obese had higher systolic BP and prevalence of metabolic syndrome than the nonsarcopenic obese group. Meanwhile, the sarcopenic nonobese group showed higher levels of total cholesterol, LDLC, systolic BP, and prevalence of metabolic syndrome than the nonsarcopenic nonobese group; these findings suggest that, regardless of obesity status, sarcopenia was associated with higher systolic BP and prevalence of metabolic syndrome. Within the sarcopenic obese group, $14.2 \%$

Table 2. Characteristics of the study population according to sarcopenia and obesity

\begin{tabular}{|c|c|c|c|c|c|c|}
\hline Variable & $\begin{array}{l}\text { Sarcopenic } \\
\text { obese }\end{array}$ & $\begin{array}{c}\text { Nonsarcopenic } \\
\text { obese }\end{array}$ & P-value* & $\begin{array}{c}\text { Sarcopenic } \\
\text { nonobese }\end{array}$ & $\begin{array}{c}\text { Nonsarcopenic } \\
\text { nonobese }\end{array}$ & P-value* \\
\hline Total & $230(8.6)$ & $2,134(91.4)$ & $<0.001$ & $89(1.2)$ & $4,911(98.8)$ & $<0.001$ \\
\hline $\begin{array}{l}\text { Age (y) } \\
20-39 \\
40-59 \\
\geq 60\end{array}$ & $\begin{array}{c}54.21 \pm 1.22 \\
25(20.6) \\
71(39.3) \\
134(40.1)\end{array}$ & $\begin{array}{c}45.72 \pm 0.49 \\
570(35.5) \\
920(46.2) \\
644(18.4)\end{array}$ & $<0.001$ & $\begin{array}{c}56.42 \pm 2.01 \\
14(18.1) \\
28(40.7) \\
47(41.2)\end{array}$ & $\begin{array}{l}43.59 \pm 0.40 \\
1,732(44.0) \\
1,880(40.0) \\
1,299(16.1)\end{array}$ & $<0.001$ \\
\hline Body height (cm) & $159.29 \pm 0.74$ & $165.06 \pm 0.28$ & $<0.001$ & $156.82 \pm 0.95$ & $164.19 \pm 0.18$ & $<0.001$ \\
\hline Body weight (kg) & $74.78 \pm 1.32$ & $74.91 \pm 0.34$ & 0.923 & $56.75 \pm 0.83$ & $59.06 \pm 0.16$ & 0.006 \\
\hline Body mass index $\left(\mathrm{kg} / \mathrm{cm}^{2}\right)$ & $29.31 \pm 0.34$ & $27.40 \pm 0.08$ & $<0.001$ & $23.03 \pm 0.17$ & $21.83 \pm 0.04$ & $<0.001$ \\
\hline Waist circumference (cm) & $96.11 \pm 0.82$ & $90.24 \pm 0.24$ & $<0.001$ & $80.76 \pm 0.70$ & $76.48 \pm 0.17$ & $<0.001$ \\
\hline ASM (kg) & $16.89 \pm 0.41$ & $21.25 \pm 0.15$ & $<0.001$ & $12.38 \pm 0.31$ & $17.45 \pm 0.09$ & $<0.001$ \\
\hline ASM/body weight (\%) & $22.34 \pm 0.22$ & $28.10 \pm 0.11$ & $<0.001$ & $21.64 \pm 0.28$ & $29.26 \pm 0.10$ & $<0.001$ \\
\hline Fasting plasma glucose (mg/dL) & $107.47 \pm 2.46$ & $100.89 \pm 0.72$ & 0.012 & $98.63 \pm 3.14$ & $93.91 \pm 0.39$ & 0.136 \\
\hline Total cholesterol (mg/dL) & $200.87 \pm 3.10$ & $196.28 \pm 0.96$ & 0.147 & $196.25 \pm 3.89$ & $182.93 \pm 0.64$ & 0.001 \\
\hline Triglyceride (mg/dL) & $170.61 \pm 9.57$ & $167.68 \pm 3.91$ & 0.777 & $127.42 \pm 9.37$ & $116.63 \pm 1.90$ & 0.266 \\
\hline High density lipoprotein cholesterol (mg/dL) & $45.77 \pm 0.88$ & $44.82 \pm 0.26$ & 0.304 & $49.61 \pm 1.86$ & $50.36 \pm 0.22$ & 0.687 \\
\hline Low density lipoprotein cholesterol (mg/dL) & $120.98 \pm 2.83$ & $117.92 \pm 0.97$ & 0.284 & $121.16 \pm 3.12$ & $109.24 \pm 0.57$ & $<0.001$ \\
\hline Systolic BP (mm Hg) & $129.28 \pm 1.44$ & $123.39 \pm 0.50$ & $<0.001$ & $122.10 \pm 2.14$ & $116.14 \pm 0.39$ & 0.006 \\
\hline Diastolic BP (mm Hg) & $81.38 \pm 0.98$ & $80.65 \pm 0.36$ & 0.481 & $76.53 \pm 1.12$ & $75.39 \pm 0.26$ & 0.320 \\
\hline Metabolic syndrome & $167(66.4)$ & $1,158(49.2)$ & $<0.001$ & $28(32.2)$ & $858(14.7)$ & $<0.001$ \\
\hline Depression status & & & 0.273 & & & 0.353 \\
\hline Not depressed & $191(81.2)$ & $1,821(85.9)$ & & $75(80.8)$ & 4,206 (85.6) & \\
\hline Depressed & 27 (14.2) & $235(10.5)$ & & $9(12.2)$ & $522(11.0)$ & \\
\hline Depression & $12(4.6)$ & 78 (3.6) & & $5(7.0)$ & $183(3.5)$ & \\
\hline
\end{tabular}

Values are presented as mean \pm standard error of number (\%).

ASM, appendicular skeletal muscle mass; BP, blood pressure.

${ }^{*}$ Calculated by analysis of variance and chi-square tests. 
Table 3. Comparisons of depressive states and sarcopenia by age

\begin{tabular}{|c|c|c|c|c|c|c|}
\hline Variable & Sarcopenic obese & Nonsarcopenic obese & P-value* & Sarcopenic nonobese & Nonsarcopenic nonobese & P-value ${ }^{*}$ \\
\hline \multicolumn{7}{|c|}{ Not depressed (y) } \\
\hline All age & 191 (81.2) & $1,821(85.9)$ & 0.273 & 75 (80.8) & 4,206 (85.6) & 0.353 \\
\hline $20-39$ & 23 (89.5) & $502(88.7)$ & 0.597 & $11(80.7)$ & $1,524(87.0)$ & 0.571 \\
\hline $40-59$ & 58 (81.6) & 776 (84.6) & 0.514 & $22(71.7)$ & $1,597(84.9)$ & 0.271 \\
\hline$\geq 60$ & $110(76.5)$ & $543(84.0)$ & 0.164 & $42(89.9)$ & $1,085(83.4)$ & 0.140 \\
\hline \multicolumn{7}{|c|}{ Depressed (y) } \\
\hline All age & $27(14.2)$ & 235 (10.5) & 0.273 & $9(12.2)$ & $522(11.0)$ & 0.353 \\
\hline 20-39 & $2(10.5)$ & $51(7.6)$ & 0.597 & $2(11.9)$ & 159 (10.2) & 0.571 \\
\hline $40-59$ & $7(11.8)$ & $108(12.0)$ & 0.514 & $4(21.9)$ & $212(11.3)$ & 0.271 \\
\hline$\geq 60$ & $18(18.6)$ & $76(12.1)$ & 0.164 & $3(2.8)$ & $151(12.2)$ & 0.140 \\
\hline \multicolumn{7}{|c|}{ Depression (y) } \\
\hline All age & $12(4.6)$ & $78(3.6)$ & 0.273 & $5(7.0)$ & $183(3.5)$ & 0.353 \\
\hline 20-39 & 0 & $17(3.7)$ & 0.597 & $1(7.4)$ & $49(2.8)$ & 0.571 \\
\hline $40-59$ & $6(6.6)$ & $36(3.4)$ & 0.514 & $2(6.4)$ & $71(3.8)$ & 0.271 \\
\hline$\geq 60$ & $6(4.9)$ & $25(3.9)$ & 0.164 & $2(7.3)$ & $63(4.5)$ & 0.140 \\
\hline
\end{tabular}

Values are presented as number (\%).

${ }^{*}$ Calculated by analysis of variance and chi-square test, and layered analysis.

Table 4. Adjusted ORs for sarcopenia

\begin{tabular}{|c|c|c|c|c|}
\hline Variable & $\begin{array}{l}\text { Unadjusted ORs } \\
\qquad(95 \% \mathrm{Cl})\end{array}$ & P-value* & $\begin{array}{l}\text { Adjusted ORs } \\
\quad(95 \% \mathrm{Cl})\end{array}$ & P-value ${ }^{*}$ \\
\hline $\begin{array}{l}\text { Total }^{\dagger} \\
\text { Depressed } \\
\text { Depression }\end{array}$ & $\begin{array}{l}1.346(0.866-2.091) \\
1.538(0.867-2.725)\end{array}$ & 0.160 & $\begin{array}{l}1.088(0.682-1.736) \\
1.116(0.585-2.128)\end{array}$ & 0.897 \\
\hline $\begin{array}{l}\text { Men }^{\dagger} \\
\text { Depressed } \\
\text { Depression }\end{array}$ & $\begin{array}{l}1.721(0.618-4.793) \\
2.306(0.737-7.216)\end{array}$ & 0.251 & $\begin{array}{l}1.618(0.574-4.558) \\
1.760(0.467-6.638)\end{array}$ & 0.503 \\
\hline $\begin{array}{l}\text { Women }^{\ddagger} \\
\text { Depressed } \\
\text { Depression }\end{array}$ & $\begin{array}{l}1.063(0.662-1.707) \\
1.133(0.586-2.190)\end{array}$ & 0.901 & $\begin{array}{l}0.915(0.572-1.465) \\
0.905(0.427-1.916)\end{array}$ & 0.900 \\
\hline
\end{tabular}

$\mathrm{OR}$, odds ratio; $\mathrm{Cl}$, confidence interval; $\mathrm{BMI}$, body mass index.

${ }^{*}$ Calculated by multiple regression analysis. ${ }^{\dagger}$ Total population and men were adjusted for sex, age, BMl, exercise, smoking, metabolic syndrome, diabetes mellitus, and hypertension. ${ }^{\ddagger}$ Women were adjusted for sex, age, BMl, exercise, smoking, metabolic syndrome, diabetes mellitus, hypertension, and menopause.

had depressed symptoms, and $4.6 \%$ were depressed, compared to $10.5 \%$ and $3.6 \%$, respectively, in the nonsarcopenic obese group. These results demonstrate that depressed symptoms and depression were higher in obese participants when accompanied by sarcopenia; however, these findings were not statistically significant. Similarly, depressive symptoms and prevalence of depression were higher in the sarcopenic nonobese group than in the nonsarcopenic nonobese group, again without statistical significance (Table 2).

\section{Sarcopenia and Depression by Age Group}

Stratified analysis was performed by age group after dividing the study population into four groups by sarcopenia and obesity status. Sarcopenia was not associated with depressive symptoms or prevalence of depression in any age group (20-39, 4059 , and $\geq 60$ years) (Table 3 ).

\section{Depression according to Sarcopenia Status}

Multiple logistic regression analysis showed no association be- tween sarcopenia and depression before and after adjusting for covariates. Moreover, sarcopenia was not associated with depression in either men or women (Table 4).

\section{DISCUSSION}

The present study used KNHANES V-1, 2 data to investigate the association between sarcopenia and depression. Although the sarcopenia group showed higher depressive symptoms and prevalence of depression than the nonsarcopenia group, the findings were not statistically significant. Sarcopenia was not associated with depression even in analyses that considered obesity status, age, and gender.

This study had several limitations. First, subjective judgment may influence the depression-related survey process. Koreans have a tendency to respond negatively to mental health-related questions. This tendency can lead to underestimation of the prevalence of depressive symptoms and depression, which may limit the informativeness of statistical testing. Second, classification errors are also possible since survey questions, rather than a specialized depression assessment tool, were used for classification. The relationship between two factors, therefore, might be underestimated due to the influence of non-discriminatory classification errors.

Previous studies have mostly reported associations between sarcopenia and depression in elderly men. Lee et al. ${ }^{23)}$ reported that a decrease in skeletal muscles was associated with depressive symptoms in elderly Chinese men, while Kim et al. ${ }^{15)}$ reported that sarcopenia was associated with depression in elderly Korean men. A study by Hsu et al. ${ }^{16)}$ also reported sarcopenia to be associated with depression in Taiwanese veterans.

Although the mechanism of association between sarcopenia and depression has not been clearly identified, many factors have been suggested. Chronic low-level inflammation, ${ }^{24,25)}$ ex- 
cessive oxidative stress, ${ }^{26,27)}$ and testosterone ${ }^{28,29)}$ may be predictive factors for these diseases.

Unlike previous studies, the findings in the present study showed no association between sarcopenia and depression. While sarcopenia and depression share common factors, they have a weak influence on the association between these diseases. The typical physical changes in the elderly involve body composition changes, including increased body fat mass and weakening of the musculoskeletal system. The association between obesity, which is characteristic of body fat mass increase, and depression has been reported; however, the association between sarcopenia and depression remains inconsistent. Therefore, while the pathophysiology of depression is likely associated with an increase in body fat mass and adipose tissues, its association with a skeletal muscle decrease requires additional study. The scarcity of other studies on this topic, therefore, make the findings of the present study important for determining the criteria for associations between sarcopenia and other diseases.

The present study has several limitations. First, muscle strength or function was not considered when diagnosing sarcopenia. The diagnosis of sarcopenia based only on decreased skeletal muscle mass used in the present study may have resulted in errors in identifying participants with sarcopenia. Second, the cross-sectional nature of this study limited its ability to prove a causal relationship between sarcopenia and depression. Therefore, future longitudinal studies, including large-scale cohort studies, are necessary. Third, the use of a subjective questionnaire rather than a depression assessment tool to define the depressed state in this study could have led to underestimation and classification errors.

Despite these limitations, the present study is significant from the perspective that it used nationally representative data from KNHANES-V. The fact that the findings were contrary to previous studies is also meaningful. Future large-scale cohort studies on the association between sarcopenia and depression, as well as studies on the pathophysiology of sarcopenia, are necessary.

\section{CONFLICT OF INTEREST}

No potential conflict of interest relevant to this article was reported.

\section{REFERENCES}

1. Peveler R, Carson A, Rodin G. Depression in medical patients. BMJ 2002;325:149-52.

2. Shim RS, Baltrus P, Ye J, Rust G. Prevalence, treatment, and control of depressive symptoms in the United States: results from the National Health and Nutrition Examination Survey (NHANES), 2005-2008. J Am Board Fam Med 2011;24:33-8.
3. Bromet E, Andrade LH, Hwang I, Sampson NA, Alonso J, de Girolamo $\mathrm{G}$, et al. Cross-national epidemiology of DSM-IV major depressive episode. BMC Med 2011;9:90.

4. Seoul National University College of Medicine. The epidemiological survey of mental disorders in Korea 2011. Sejong: Ministry of Health and Welfare; 2012.

5. Chang SM, Hahm BJ, Lee JY, Shin MS, Jeon HJ, Hong JP, et al. Cross-national difference in the prevalence of depression caused by the diagnostic threshold. J Affect Disord 2008;106:159-67.

6. Rosenberg IH. Sarcopenia: origins and clinical relevance. J Nutr 1997; 127(5 Suppl):990S-991S.

7. Baumgartner RN, Koehler KM, Gallagher D, Romero L, Heymsfield SB, Ross RR, et al. Epidemiology of sarcopenia among the elderly in New Mexico. Am J Epidemiol 1998;147:755-63.

8. Bijlsma AY, Meskers CG, Westendorp RG, Maier AB. Chronology of agerelated disease definitions: osteoporosis and sarcopenia. Ageing Res Rev 2012;11:320-4.

9. Janssen I, Shepard DS, Katzmarzyk PT, Roubenoff R. The healthcare costs of sarcopenia in the United States. J Am Geriatr Soc 2004;52:80-5.

10. Janssen I, Heymsfield SB, Ross R. Low relative skeletal muscle mass (sarcopenia) in older persons is associated with functional impairment and physical disability. J Am Geriatr Soc 2002;50:889-96.

11. Stenholm S, Harris TB, Rantanen T, Visser M, Kritchevsky SB, Ferrucci L. Sarcopenic obesity: definition, cause and consequences. Curr Opin Clin Nutr Metab Care 2008;11:693-700.

12. Cole MG, Dendukuri N. Risk factors for depression among elderly community subjects: a systematic review and meta-analysis. Am J Psychiatry 2003;160:1147-56.

13. Luppino FS, de Wit LM, Bouvy PF, Stijnen T, Cuijpers P, Penninx BW, et al. Overweight, obesity, and depression: a systematic review and metaanalysis of longitudinal studies. Arch Gen Psychiatry 2010;67:220-9.

14. Cho Y, Shin SY, Shin MJ. Sarcopenic obesity is associated with lower indicators of psychological health and quality of life in Koreans. Nutr Res 2015;35:384-92.

15. Kim NH, Kim HS, Eun CR, Seo JA, Cho HJ, Kim SG, et al. Depression is associated with sarcopenia, not central obesity, in elderly korean men. J Am Geriatr Soc 2011;59:2062-8.

16. Hsu YH, Liang CK, Chou MY, Liao MC, Lin YT, Chen LK, et al. Association of cognitive impairment, depressive symptoms and sarcopenia among healthy older men in the veterans retirement community in southern Taiwan: a cross-sectional study. Geriatr Gerontol Int 2014;14 Suppl 1:102-8.

17. Korea Centers for Disease Control and Prevention. The fifth Korea National Health and Nutrition Examination Survey (KNHANES V-1, 2) 2010-2011. Cheongju: Korea Centers for Disease Control and Prevention; 2010-2011.

18. Koo S, Park K. Associations of serum 25(OH)D levels with depression and depressed condition in Korean adults: results from KNHANES 2008-2010. J Nutr Health 2014;47:113-23.

19. Muscaritoli M, Anker SD, Argiles J, Aversa Z, Bauer JM, Biolo G, et al. Consensus definition of sarcopenia, cachexia and pre-cachexia: joint document elaborated by Special Interest Groups (SIG) "cachexia-anorexia in chronic wasting diseases" and "nutrition in geriatrics". Clin Nutr 2010;29:154-9.

20. Moon SS. Low skeletal muscle mass is associated with insulin resistance, 
diabetes, and metabolic syndrome in the Korean population: the Korea National Health and Nutrition Examination Survey (KNHANES) 2009-2010. Endocr J 2014;61:61-70.

21. Grundy SM, Cleeman JI, Daniels SR, Donato KA, Eckel RH, Franklin BA, et al. Diagnosis and management of the metabolic syndrome: an American Heart Association/National Heart, Lung, and Blood Institute Scientific Statement. Circulation 2005;112:2735-52.

22. Lee SY, Park HS, Kim DJ, Han JH, Kim SM, Cho GJ, et al. Appropriate waist circumference cutoff points for central obesity in Korean adults. Diabetes Res Clin Pract 2007;75:72-80.

23. Lee JS, Auyeung TW, Kwok T, Lau EM, Leung PC, Woo J. Associated factors and health impact of sarcopenia in older chinese men and women: a cross-sectional study. Gerontology 2007;53:404-10.

24. Jensen GL. Inflammation: roles in aging and sarcopenia. JPEN J Parenter Enteral Nutr 2008;32:656-9.
25. Baune BT, Smith E, Reppermund S, Air T, Samaras K, Lux O, et al. Inflammatory biomarkers predict depressive, but not anxiety symptoms during aging: the prospective Sydney Memory and Aging Study. Psychoneuroendocrinology 2012;37:1521-30.

26. Meng SJ, Yu LJ. Oxidative stress, molecular inflammation and sarcopenia. Int J Mol Sci 2010;11:1509-26.

27. Bakunina N, Pariante CM, Zunszain PA. Immune mechanisms linked to depression via oxidative stress and neuroprogression. Immunology 2015 Jan 10 [Epub]. http://dx.doi.org/10.1111/imm.12443.

28. Maggio M, Lauretani F, Ceda GP. Sex hormones and sarcopenia in older persons. Curr Opin Clin Nutr Metab Care 2013;16:3-13.

29. Zarrouf FA, Artz S, Griffith J, Sirbu C, Kommor M. Testosterone and depression: systematic review and meta-analysis. J Psychiatr Pract 2009; 15:289-305. 